\title{
SOBRE LA COMPRENSIÓN EN ESTUDIANTES DE MATEMÁTICAS DEL CONCEPTO DE INTEGRAL IMPROPIA. ALGUNAS DIFICULTADES, OBSTÁCULOS Y ERRORES
}

\author{
González-Martín, Alejandro S. y Camacho Machín, Matías \\ Departamento de Análisis Matemático. Universidad de La Laguna \\ asglez@ull.es \\ mcamacho@ull.es
}

\begin{abstract}
Resumen. En el presente trabajo mostramos algunas de las dificultades, obstáculos y errores que los alumnos universitarios encuentran al aprender los conceptos relativos a la integración impropia; algunos de ellos parecen inherentes al propio concepto de integral impropia y otros vienen relacionados con ausencia de significado o con otros conceptos del cálculo.

Con el objetivo de analizar estas dificultades, obstáculos y errores construimos un marco teórico basado, principalmente, en la teoría de Duval sobre los registros semióticos de representación y construimos un modelo de competencia para evaluar la comprensión de nuestros alumnos.

Palabras clave. Integral impropia, registro de representación semiótica, modelo de competencia, integral definida.
\end{abstract}

Summary. In this paper we present some difficulties, obstacles and errors university students face when learning the concepts relative to improper integration. Some of them seem to be inherent to the improper integral concept itself and some others are related to lack of meaning or to other concepts of calculus.

With the aim of analysing these difficulties, obstacles and errors we built up a theoretical framework based, fundamentally, on Duval's theory of semiotic registers of representation and we designed a competence model to assess our students' comprehension.

Keywords. Improper integral, semiotic registrer of representation, competence model, definitive integral.

\section{INTRODUCCIÓN Y ANTECEDENTES}

Presentamos en este artículo algunos de los resultados de un trabajo realizado con el objetivo principal de localizar dificultades, obstáculos y errores que aparecen en los alumnos de primer año de licenciatura ${ }^{1}$ al aprender los conceptos de la integración impropia. Este trabajo representa un primer acercamiento al análisis cognitivo que se realizará para diseñar una ingeniería didáctica sobre el concepto de integral impropia, en cuyo desarrollo nos encontramos actualmente inmersos.

Es sabido que para definir la integral de Riemann² se imponen dos condiciones: intervalo de integración acotado $\mathrm{y}$ función integrando acotada en este intervalo. Estas condiciones, además, aunque son necesarias para definir esta integral no son suficientes por sí solas para asegurar la integrabilidad de una función ${ }^{3}$.

Cuando no se cumple una de las dos condiciones anteriores puede, sin embargo, extenderse la definición anterior, dando así lugar a las integrales impropias o integrales generalizadas. Uno de los problemas principales de estas integrales reside en el estudio de su convergencia. En la enseñanza universitaria tradicional se centra la atención en la memorización de criterios y en la resolución de múltiples ejercicios; sin embargo, casi no se interpretan los resultados obtenidos (y las paradojas que pueden aparecer) ni se buscan contraejemplos patológicos (en González-Martín, 2002, se realiza un análisis más detallado de los contenidos habituales en un tema de integración impropia). 
El concepto de integral impropia resulta de gran importancia para los alumnos de las titulaciones de matemáticas e ingenierías por la gran cantidad de aplicaciones que tiene, ya sea para el cálculo de probabilidades, para definir normas funcionales, en el cálculo de transformadas de Fourier y Laplace y muchos cálculos físicos (como el trabajo, la energía... en determinadas circunstancias).

A pesar de esto, nuestra experiencia nos indica que los alumnos no llegan a comprender adecuadamente este concepto ni a relacionarlo con algunos conocimientos previamente estudiados (como sucesiones, series e integrales definidas) en su primer año en la universidad, en el que toman contacto por primera vez con él. Hemos observado que muchos de los alumnos aprenden las herramientas y conceptos referentes a la integración impropia descontextualizados y desvinculados de los contenidos anteriores; se limitan a memorizar un conjunto de criterios y técnicas que, de estar contextualizados, tendrían mucho más significado para ellos.

Una de las principales dificultades que encontramos al planificar este estudio es la ausencia de trabajos publicados centrados en el concepto de integral impropia y su enseñanza-aprendizaje. Aunque éste aparece implícitamente en varios trabajos, se desarrolla generalmente en el marco de la integral definida.

Orton (1983) detecta que la introducción de los alumnos a la integración se ve oscurecida por la manipulación algebraica. En su investigación se pide calcular la integral de la función $1 / x^{2}$ en el intervalo $[-1,2]$ y se adjunta la gráfica. Esta pregunta ofreció especiales dificultades a los alumnos participantes, aunque debemos aclarar que éstos sólo tenían conocimientos elementales sobre la integración. Calvo (1997) destaca el hecho de que, al ilustrar la definición de integral definida, se suele presentar una curva sin patologías, un intervalo positivo, se usa un número razonable de rectángulos... pero no se insiste en cuáles de estos elementos son esenciales y cuáles no. Relacionado con este hecho, nosotros encontramos que nuestros alumnos no saben cuáles son las condiciones necesarias para definir la integral de Riemann.

Además, el hecho de identificar la integral como un área puede dar lugar a falsas adscripciones de significado, de forma que los alumnos no diferencian el significado de la integral cuando la función es positiva y cuando la función es negativa. Por otro lado, Schneider (1991) identifica varios errores cometidos por estudiantes en cálculos de áreas y volúmenes, que muestran la presencia de concepciones erróneas. En su trabajo identifica el obstáculo de heterogeneidad de las dimensiones, al que haremos referencia más adelante, el cual induce a mezclar cantidades de dimensiones distintas (volúmenes con áreas y áreas con líneas). Las evidencias mostradas indican que este obstáculo tiene corte epistemológico.

Rasslan y Tall (2002) diseñaron un cuestionario para explorar los esquemas cognitivos que evocan los alumnos para el concepto de integral definida, para lo cual analizaron si los estudiantes sabían definir este concepto ${ }^{4}$. Afirman que sólo 7 estudiantes de los 41 participantes dieron una definición del concepto 5 . En su cuestionario también intro- ducen dos integrales impropias, pero los alumnos no las identifican al no conocer los aspectos teóricos.

Finalmente, mencionamos el estudio de Camacho y Aguirre (2001), que plantean algunas integrales impropias a un grupo de alumnos y profesores; comprueban que tienen grandes dificultades para calcular sus valores y muchos se pierden en los cálculos algebraicos. Se observa también que tanto profesores como alumnos tienden a sustituir los valores extremos de la integral y no calculan las integrales utilizando procesos límite, sino generalizando la regla de Barrow y sustituyendo directamente los extremos de la integral en la variable, aun cuando uno de los extremos sea infinito.

Motivados por esta situación nos interesamos en esta aproximación cognitiva por estudiar cómo aprenden los alumnos de primer curso de la titulación de Matemáticas los conceptos relacionados con la integración impropia, además de determinar algunas dificultades, obstáculos y errores que surgen en su aprendizaje. Con este fin se decidió utilizar problemas no rutinarios ${ }^{6} \mathrm{y}$ de tipo no algorítmico para averiguar su nivel de comprensión, entendiendo la comprensión de un concepto matemático bajo la óptica de los sistemas de representación semiótica. Uno de nuestros objetivos es analizar el sistema de representación en el que los alumnos trabajan, además de observar si hacen alguna interpretación gráfica de los resultados que obtienen.

También nos propusimos analizar, entre otras, las siguientes cuestiones: ¿Utilizan los alumnos elementos intuitivos? ¿Articulan diferentes sistemas de representación en situaciones relativas a integrales impropias? ¿Realizan procesos de transferencia de otros conceptos previos a los nuevos?

Razones de tiempo, espacio y planificación nos llevan a acotar nuestro campo de investigación inicial y a decantarnos solamente por el uso de los registros de representación gráfico y algebraico en un primer acercamiento al análisis de la comprensión de los alumnos de algunos conceptos básicos de la integración impropia, bajo la hipótesis de que los alumnos sólo están acostumbrados al trabajo en uno de ellos (el algebraico). Esta delimitación nos permitió reducir el campo de estudio, simplificando la cantidad de hechos a observar.

Ponemos énfasis en el hecho de que, a pesar de encontrarnos elaborando una ingeniería didáctica sobre el concepto de integral impropia, los datos aquí presentados abordan una problemática de aprendizaje y no de enseñanza. En este aspecto, el diseño de un modelo de competencia cognitivo para caracterizar el nivel de comprensión de los alumnos de los procesos bajo estudio facilita enormemente el estudio cualitativo que se pretende desarrollar.

Lo explicitado anteriormente se concreta en los siguientes objetivos:

- Analizar el trabajo que desarrollan los alumnos de primer curso de la licenciatura en Matemáticas de nuestra universidad cuando se ponen en juego dos registros de representación semiótica (el algebraico y el gráfico) en tareas relativas a la integración impropia. 
- Elaborar un instrumento que, a partir del empleo de dos registros de representación semiótica, nos permita indagar en los conocimientos que tienen los alumnos sobre el concepto de integral impropia después de recibir una instrucción tradicional.

- Caracterizar los distintos niveles de comprensión del concepto de integral impropia mediante un modelo de competencia.

- Identificar dificultades, obstáculos y errores que surgen en los alumnos cuando aprenden los elementos de la integración impropia.

- Evaluar el modelo de competencia utilizado.

Para tratar de ofrecer alguna respuesta a estas preguntas se elaboró un marco teórico para evaluar los niveles de comprensión de los alumnos cuando entran en juego los sistemas de representación algebraico y gráfico. Con ayuda de este marco teórico construimos un modelo de competencia con el que poder clasificar los niveles de comprensión cuando sólo entran en juego dos sistemas semióticos de representación.

\section{MARCO TEÓRICO DE LA INVESTIGACIÓN}

\subsection{Bases del marco teórico}

Como se señala en el apartado anterior, en el planteamiento de este análisis cognitivo estamos más interesados en los fenómenos ligados al aprendizaje de la integral impropia que en un problema de enseñanza. Además, nos centramos específicamente en las actividades que realizan los alumnos para conjugar dos sistemas de representación, el gráfico y el algebraico, ligados al concepto de integral impropia. Por tanto, se ha elegido una aproximación semiótica a esta problemática, entre todas las posibles. Por otro lado, al no abordar sino el uso de dos registros, no se pretende modelizar completamente la comprensión de la integral impropia.

Tal y como señala Duval (1993, 1995), consideramos que la distinción entre un objeto matemático y su representación es fundamental para la comprensión de las matemáticas. El uso de las diferentes representaciones semióticas de un objeto matemático es, desde esta perspectiva, imprescindible para conseguir este propósito.

Bajo este enfoque, estamos interesados en localizar dificultades, obstáculos y errores que surgen de forma natural al realizar la conversión entre dos registros o al realizar manipulaciones dentro de uno de ellos.

Al considerar fenómenos ligados al aprendizaje de las matemáticas, es necesario apoyarnos en una teoría del aprendizaje para explicar estos fenómenos. En lo que sigue, se expondrá de forma resumida los supuestos teóricos bajo los cuales se ha realizado este análisis cog- nitivo (desarrollado extensamente en González-Martín, 2002); se trata de determinar un modelo de competencia cognitivo, para lo que se adaptó la propuesta que presenta Socas (2001) en relación con el lenguaje algebraico, organizada en torno a los siguientes componentes:

a) estadios de desarrollo cognitivo de los sistemas de representación;

b) la teoría de Duval sobre los registros de representación semiótica y el funcionamiento cognitivo del pensamiento;

c) dificultades, obstáculos y errores en el aprendizaje.

\section{Estadios de desarrollo cognitivo}

En el proceso en que se aprende a manejar correctamente un sistema de representación semiótico formal, se pueden distinguir varios estadios de desarrollo cognitivo. Nosotros optaremos por la siguiente organización de estos estadios como sigue: estadio semiótico, estadio estructural y estadio autónomo (Socas, 2001).

Como ya se ha dicho, al sólo interesarnos por las conversiones entre los sistemas algebraico (formal) y gráfico, no pretendemos modelizar completamente la comprensión de las integrales impropias. Más bien, los niveles de nuestro modelo de competencia se referirán a los procesos de conversión de estos dos registros y a los procesos de transferencia asociados. Esto quedará explícito más adelante.

\section{Teoría de Duval sobre los registros de representación semiótica}

Una definición de lo que entendemos por las representaciones semióticas nos la da Duval (1993):

«Las representaciones mentales cubren al conjunto de imágenes y, globalmente, a las concepciones que un individuo puede tener sobre un objeto, sobre una situación y sobre lo que les está asociado.

»Las representaciones semióticas son producciones constituidas por el empleo de signos que pertenecen a un sistema de representación, el cual tiene sus propias restricciones de significado y de funcionamiento.

»Una figura geométrica, un enunciado en lengua natural, una fórmula algebraica, una gráfica, son representaciones semióticas que pertenecen a sistemas semióticos diferentes.»

Duval continúa profundizando sobre esta idea hasta llegar a caracterizar un registro de representación de la forma que sigue:

«Un sistema semiótico puede ser un registro de representación si permite tres actividades cognitivas relacionadas con la semiosis?

»1) La formación de una representación identificable como una representación de un registro dado.

»2) El tratamiento de una representación, que es la 
transformación de la representación dentro del mismo registro donde ha sido formada. El tratamiento es una transformación interna a un registro.

»3) La conversión de una representación, que es la transformación de la representación en otra representación de otro registro en la que se conserva la totalidad o parte del significado de la representación inicial. La conversión es una transformación externa al registro de partida.

Duval enuncia que, al ser cada representación parcial con respecto a lo que representa, se debe considerar como absolutamente necesario la interacción entre diferentes representaciones para la formación del concepto. Se puede decir, entonces, que un concepto se va construyendo mediante tareas que impliquen la utilización de diferentes sistemas de representación y promuevan la articulación coherente entre representaciones. Desde esta orientación teórica, el conocimiento de un individuo sobre un concepto es estable si es capaz de articular diferentes representaciones del concepto libre de contradicciones.

Diversos autores, como Hitt (2000a), consideran que no sólo son importantes las tareas de transformación dentro de un registro de representación y las de conversión entre registros, sino que también aparece como importante la confrontación entre ejemplos y contraejemplos. Por otro lado, el trabajo de Duval no menciona los aspectos relativos al papel de las representaciones en la resolución de problemas. Bajo estos aspectos teóricos, cobra gran importancia la noción de transferencia, que se muestra esencial en el terreno de la resolución de problemas.

Para evaluar los procesos de transferencia ideamos algunas preguntas donde se utilizan expresamente conocimientos anteriores, pero en un contexto nuevo. Intentamos con esto estudiar cómo realizan los alumnos las transferencias necesarias para solucionar las nuevas cuestiones (en caso de abordarlas).

\section{Dificultades, obstáculos y errores}

Socas (1997) distingue cuatro elementos básicos como productores de dificultades en el currículo de matemáticas: las habilidades necesarias para desarrollar capacidades matemáticas que definen la competencia de un alumno en matemáticas, la necesidad de contenidos anteriores, el nivel de abstracción requerido y la naturaleza lógica de las matemáticas escolares. De forma detallada, éstas pueden ser organizadas de la siguiente forma:

- Dificultades asociadas a la complejidad de los objetos de las matemáticas.

- Dificultades asociadas a los procesos de pensamiento matemático.

- Dificultades asociadas a los procesos de enseñanza desarrollados para el aprendizaje de las matemáticas.

- Dificultades asociadas a los procesos de desarrollo cognitivo de los alumnos.

- Dificultades asociadas a actitudes afectivas y emocionales hacia las matemáticas.
Las dos primeras se relacionan con la propia disciplina, la tercera con los procesos de enseñanza, la cuarta con los procesos cognitivos de los alumnos y la quinta con el terreno afectivo.

Estas dificultades se relacionan y forman redes en las que se refuerzan, concretándose en la práctica en forma de obstáculos y manifestándose en forma de errores. Socas (1997) proporciona también algunas características de los obstáculos:

- Un obstáculo es un conocimiento adquirido, no una falta de conocimiento.

- Tiene un dominio de eficacia y se utiliza para producir respuestas adaptadas a un cierto contexto.

- Cuando este conocimiento se utiliza fuera de su contexto, genera respuestas inadecuadas, incluso incorrectas.

- Es resistente y resultará más resistente cuanto mejor adquirido esté o cuanto más haya demostrado su eficacia y su potencia en el anterior dominio de validez.

- Después de haber notado su inexactitud, continúa manifestándose esporádicamente.

Con frecuencia los errores se manifiestan durante la manipulación de una representación dentro de un mismo sistema de representación, que generalmente es el algebraico. Otro tipo de error se puede presentar cuando hay una elección inadecuada de un sistema semiótico al resolver un problema matemático. También, como señala Duval (1993), muchas de las dificultades encontradas por los estudiantes en diferentes niveles del currículo pueden ser descritas y explicadas como una falta de coordinación de registros de representación. Además, la construcción inadecuada de un concepto se puede deber a una carencia de articulación entre diferentes registros semióticos de representación.

La complejidad de las dificultades del aprendizaje de las matemáticas se traduce en errores que cometen los alumnos, que se producen por causas muy diversas. Es necesario, desde los modelos de competencia, tener elementos de análisis de estos errores; pero este análisis no se puede hacer en forma aislada, sino mediante actividades que intenten esclarecer las posibles conexiones (articulaciones) realizadas por ellos durante la construcción de algún concepto dado.

\subsection{Modelo de competencia}

Desde nuestro enfoque teórico, y con los instrumentos diseñados, definimos la competencia como la articulación coherente de diferentes registros semióticos; ser competente significaría articular coherentemente las diferentes representaciones de la integral impropia al enfrentarse a la resolución de problemas «no rutinarios».

Como ya hemos comentado, hemos extendido los diferentes estadios de desarrollo que se dan en los sistemas de representación cognitivos en el caso del lenguaje algebraico (Socas, 2001) a la coordinación entre dos 
registros en nuestro caso particular. De esta forma, ya no hablaremos de niveles dentro de un mismo registro, sino de niveles en la coordinación entre dos registros ${ }^{8}$. En cada estadio distinguiremos dos categorías de comportamiento, obteniendo las siguientes categorías, que hacen referencia a las posibles articulaciones entre dos registros en cuestiones sobre integración impropia:

\begin{tabular}{|l|l|}
\hline Categoría 1A & $\begin{array}{l}\text { El alumno tiene ideas imprecisas sobre la integral } \\
\text { impropia y mezcla de forma incoherente diferentes } \\
\text { representaciones semióticas. }\end{array}$ \\
\hline Categoría 1B & $\begin{array}{l}\text { El alumno reconoce los elementos de un sistema } \\
\text { de representación semiótico en relación con la } \\
\text { integral impropia. }\end{array}$ \\
\hline Categoría 2A & $\begin{array}{l}\text { El alumno conoce un sistema de representación } \\
\text { semiótico y realiza transformaciones en el interior } \\
\text { del sistema de representación. }\end{array}$ \\
\hline Categoría 2B & $\begin{array}{l}\text { El alumno realiza correctamente actividades de } \\
\text { conversión de un sistema de representación se- } \\
\text { miótico a otro; en estas actividades de conversión } \\
\text { hay un sistema que el alumno controla, y facilita la } \\
\text { conversión al otro. }\end{array}$ \\
\hline Categoría 3A & $\begin{array}{l}\text { El alumno articula dos sistemas de representación } \\
\text { semióticos. Puede tomar cualquiera de ellos para } \\
\text { significar correctamente al objeto integral impropia } \\
\text { independientemente del otro. El alumno maneja autóno- } \\
\text { mamente los dos sistemas de representación semióticos. }\end{array}$ \\
\hline Categoría 3B & $\begin{array}{l}\text { El alumno articula coherentemente diferentes sis- } \\
\text { temas de representación semióticos, ejerce un con- } \\
\text { trol de las representaciones semióticas que utiliza. } \\
\text { Tiene conocimiento de la integral impropia como } \\
\text { estructura y puede controlar aspectos coherentes e } \\
\text { incoherentes del mismo. }\end{array}$ \\
\hline
\end{tabular}

Nosotros solamente consideramos los registros de representación gráfico y algebraico, a los que se añade necesariamente el de la lengua natural, en las cuestiones utilizadas en las entrevistas, que son los siguientes:

Representaciones en lenguaje habitual $(\mathrm{H})$ Representaciones en lenguaje formal (algebraico) $(\mathrm{F})^{9}$ Representaciones en lenguaje visual gráfico $(\mathrm{G})$

Por tanto, las acciones que distinguiremos serán:

\begin{tabular}{|l|c|}
\hline $\begin{array}{l}\text { Reconocimiento de los elementos de un sistema de } \\
\text { representación semiótico }\end{array}$ & $R_{H}, R_{F}, R_{G}$ \\
\hline $\begin{array}{l}\text { Transformaciones internas en un registro de } \\
\text { representación semiótico }\end{array}$ & $T_{H}, T_{F}, T_{G}$ \\
\hline $\begin{array}{l}\text { Conversiones (transformaciones externas) entre } \\
\text { sistemas de representación semióticos }\end{array}$ & $\begin{array}{l}C_{H \rightarrow F}, C_{H \rightarrow G} \\
C_{F \rightarrow G}, C_{G \rightarrow F}\end{array}$ \\
\hline $\begin{array}{l}\text { Coordinación entre diferentes sistemas de } \\
\text { representación semióticos }\end{array}$ & $\begin{array}{c}C_{F \leftrightarrow G}, C_{G \leftrightarrow F} \\
C_{H \leftrightarrow F} \\
C_{H \leftrightarrow G}\end{array}$ \\
\hline $\begin{array}{l}\text { Producción de representaciones semióticas en la } \\
\text { resolución de una tarea }\end{array}$ & $P S_{F}, P S_{G}$ \\
\hline
\end{tabular}

Esta notación es utilizada para describir las tareas esperadas en los alumnos entrevistados en las cuestiones que se les plantearon y tratar de ubicarlos en alguna de las seis categorías de comportamiento descritas anteriormente; la valoración sobre el conocimiento de los estudiantes se analiza sobre actividades que intenten esclarecer las posibles conexiones (articulaciones) realizadas.

\section{METODOLOGÍA}

\subsection{La población}

Participan en la experiencia 31 estudiantes, de los que 13 son hombres y 18, mujeres. Los alumnos cursan asignaturas de primer año de la titulación en Matemáticas y todos están matriculados en la asignatura Análisis Matemático II, que es la asignatura donde se tratan los contenidos propios a la integración en una variable y, en particular, los relacionados con la integración impropia. Se distribuyen en dos grupos, tal como se muestra en la siguiente tabla.

\begin{tabular}{|c|c|c|c|c|}
\hline & \multicolumn{2}{|c|}{ SEXO } & \\
\hline & & Hombre & Mujer & \\
\hline \multirow{2}{*}{ 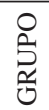 } & $1-\mathrm{A}$ & 9 & 9 & 18 \\
\hline & $1-B$ & 4 & 9 & 13 \\
\hline & & 13 & 18 & 31 \\
\hline
\end{tabular}

También sabemos que para la mayoría de los alumnos es el segundo año que cursan esta asignatura.

\subsection{Instrumentos de recogida de la información}

Conscientes del interés y la riqueza que aporta el análisis cualitativo en una investigación como ésta, desde el principio se planteó el uso de cuestionarios y entrevistas para realizar nuestro estudio. Esto se convirtió en una necesidad aún mayor después de realizar la prueba escrita y analizar las respuestas obtenidas. La información que se pudo obtener de las entrevistas grabadas a partir de los datos del cuestionario resultó mucho más rica que la que obtuvimos de la corrección de las respuestas escritas en el papel.

Por tanto, se utilizaron dos instrumentos para recoger la información: un cuestionario de conocimientos y entrevistas semiestructuradas. El cuestionario consta de nueve preguntas, incluyendo no sólo tareas de cálculo directo de integrales impropias y estudio de convergencia de integrales impropias, sino también cuestiones intuitivas y algunos resultados paradójicos. En particular, se pide interpretar la mayoría de los resultados a los que se llega (González-Martín y Camacho, 2002a, 2004; GonzálezMartín, 2002). Posteriormente se seleccionaron algunos alumnos para ser entrevistados y obtener información sobre los procesos y razonamientos que habían seguido 
en sus respuestas y las concepciones que tienen del objeto «integral impropia».

En este sentido, la necesidad de estudiar cuestiones relativas a las respuestas obtenidas inicialmente nos condujo a utilizar, como protocolo de las entrevistas, las preguntas planteadas en el cuestionario. En el apartado 6 se explica detalladamente qué preguntas se seleccionaron y con qué objetivos.

La técnica usada fue la de entrevista basada en un cuestionario $^{10}$, en la que el entrevistado no sólo interactúa con el entrevistador, sino también con el conjunto de tareas que se le encomiendan (preguntas, problemas o actividades). En nuestro caso, las entrevistas no sólo fueron audiograbadas, sino también videograbadas, ya que estábamos interesados en recoger gran cantidad de información ${ }^{11}$.

\subsection{Selección de los entrevistados}

La selección de los alumnos se hizo según sus respuestas al cuestionario. Básicamente, nos apoyamos en la combinación de los dos criterios siguientes: el desempeño total en la prueba o algunas representaciones o formas de proceder muy significativas. En total se entrevistó a 6 alumnos, lo que supone un 19,35\% del total, casi la quinta parte. Los alumnos seleccionados cumplen las siguientes características:

\begin{tabular}{|c|c|c|c|}
\hline Alumno & Grupo & Sexo & $\begin{array}{l}\text { Resultado del } \\
\text { cuestionario }^{12}\end{array}$ \\
\hline MA1 & \multirow{3}{*}{$1-A$} & \multirow{2}{*}{ Mujer } & Medio \\
\hline MA2 & & & Bajo \\
\hline MA3 & & \multirow{4}{*}{ Hombre } & Muy alto \\
\hline MA4 & \multirow{2}{*}{$1-B$} & & Alto \\
\hline MA5 & & & Muy alto \\
\hline MA6 & $1-\mathrm{A}$ & & Alto \\
\hline
\end{tabular}

Las entrevistas tuvieron una duración variable, según las respuestas de los alumnos. Estimamos que la más corta (MA5) duró alrededor de treinta y cinco minutos y que la más larga (MA1) duró una hora y veinte minutos.

De las nueve preguntas de que consta el cuestionario se seleccionaron cinco preguntas para la entrevista y se añadió una más. Para cada entrevista se llevó la prueba de conocimientos del alumno entrevistado para hacerle preguntas particulares sobre lo que había escrito y, si realizaba algún cambio, averiguar las razones. Además, también servía para ayudar a algún alumno si se quedaba bloqueado.

\section{ANÁLISIS DE LOS RESULTADOS}

A continuación mostramos las seis cuestiones del protocolo de las entrevistas junto con los objetivos propuestos con cada cuestión, comentando brevemente algunos de los resultados obtenidos en el cuestionario; formulamos las acciones esperadas según nuestro modelo de competencia, para finalmente analizar y discutir algunos datos obtenidos en la entrevista ${ }^{13}$.

\section{Pregunta 1 \\ ¿Cómo le explicarías a un compañero el significado de $\int_{a}^{b} f(x) \cdot d x$ ?}

Con esta pregunta se quiere saber si los alumnos tienen claro, o bien asimilado, el concepto del que se supone que se va a partir para construir el de integral impropia.

En el análisis de los cuestionarios se había obtenido que casi todos los alumnos la definen como un área (29 de los 31 alumnos). Sabiendo que el registro de representación gráfico va a estar muy presente en el resto de preguntas que se va a plantear, nos interesamos por saber si son coherentes con los registros que usan para definir la integral y para operar con ella.

También se observó en la prueba escrita que tan sólo 4 alumnos aluden a que la integral definida es un área sólo si la función es positiva en el intervalo de integración, y otros 4 se refieren a que el intervalo de integración sea finito. Con esta cuestión se pretende averiguar si no se ha puntualizado este detalle por mero olvido o si realmente los alumnos conciben que siempre debe ser un área.

Por último, pensamos que los alumnos se sentirían más cómodos comenzando la entrevista con una pregunta que ellos consideraran, de antemano, «fácil».

Las acciones que consideramos a priori, según nuestro modelo de competencia, son las siguientes:

\begin{tabular}{|c|c|}
\hline & $R_{H}, R_{F}$ \\
Pregunta 1 & $C_{H \rightarrow F}, C_{F \rightarrow G}$ \\
& $C_{F \leftrightarrow G}, C_{G \leftrightarrow F}$ \\
& $P S_{G}$ \\
\hline
\end{tabular}

\section{Análisis y discusión}

Un primer obstáculo que observamos consiste en pensar que la integral es siempre un área, independientemente del tipo de función; este tipo de obstáculos ya ha sido detectado en el trabajo de Calvo (1997), entre otros. La alumna MA1 explica:

A: [...] Pues que es el área comprendida... de la curva $f(x) \ldots[\ldots]$ y el eje de abscisas y las rectas $x=a$ y $x=b$.

$[\ldots]$

E: ¿Es cierto? ¿Para toda función? ¿Es siempre el área? ¿Para toda función?

A: ... Sí... 
Además, esta alumna define más adelante la integral como una «aproximación» al área. Esto puede estar unido al obstáculo citado por Tall (1992b), en el que los alumnos ven el límite como algo inalcanzable; pensamos que también puede estar relacionado con el uso del infinito potencial (Hitt, 2000b; Garbin, 1998).

El estudiante MA3 da una definición muy formal, pero concibe también la integral siempre como un área ${ }^{14}$. Este alumno aborda todas las preguntas de forma claramente algebraica; no parece realizar correctamente las coordinaciones entre los registros algebraico y gráfico $\left(C_{F \bullet G}\right.$ y $C_{G \hookleftarrow F}$ ). Resolvió todas las preguntas del cuestionario algebraicamente, apenas recurriendo a lo gráfico, por lo que fue seleccionado para saber si simplemente prefiere el registro algebraico o si tiene dificultades con el registro gráfico.

En el caso de la alumna MA2, su definición es operacional:

A: Bueno, pues la integral es eso, un modo de calcular... una forma de cálculo... de... de... de un... un área...

Pensamos que su conversión al sistema formal $\left(C_{H, F}\right)$ es incompleta, pues su misma definición de integral está a nivel de proceso. Pensamos que ve la integral como una herramienta para calcular el área (un procedimiento, quizá una operación), pero no como el área misma. Esto ya se convierte en un obstáculo para ella al abordar preguntas de corte no algorítmico. Este tipo de comportamientos ha sido presentado por varios autores (Tall, 1994), relacionado con el uso generalizado de los símbolos matemáticos de forma compresiva; muchos procesos matemáticos se simbolizan y posteriormente este símbolo se trata como un concepto matemático, pudiendo ser manipulado como un objeto mental ${ }^{15}$. Dada la gran cantidad de símbolos que representan tanto el proceso como el concepto, Tall introduce la definición de procepto elemental como la amalgama de un proceso, un concepto relativo producido por tal proceso y un símbolo que los representa a ambos.

Evidentemente, la noción de procepto conlleva una ambigüedad, pues un símbolo puede ser interpretado como un proceso o como un objeto, que lleva a la flexibilidad del pensamiento: se puede usar el proceso para hacer matemáticas y obtener respuestas o utilizar el concepto para pensar en matemáticas. Sin embargo, los alumnos que no han desarrollado esta flexibilidad son incapaces de ver el símbolo como un concepto, sino solamente como un proceso.

\section{Pregunta 2}

Sabemos que $f(x)$ es una función continua y estrictamente positiva. Supón que hemos calculado $\int_{1}^{10} f(x) d x \quad$ y que nos da una cantidad finita. Ahora calculamos $\int_{1}^{20} f(x) d x \quad$ y también nos da una cantidad finita, mayor que la anterior.

Si seguimos calculando:

$$
\int_{1}^{30} f(x) d x, \int_{1}^{40} f(x) d x, \int_{1}^{50} f(x) d x, \int_{1}^{10-n} f(x) d x \ldots
$$

¿qué crees que sucederá?

¿Podrá alguna vez converger esta sucesión? ¿Qué tipo de funciones $f(x)$ pueden dar lugar a una sucesión convergente?
Ésta es la única cuestión nueva introducida en la entrevista, con la finalidad de analizar los razonamientos de los alumnos en una situación, en principio, desconocida para ellos. Tiene como objetivo averiguar qué tipo de transferencias hacen los alumnos de sus conocimientos de sucesiones y series a los de integración impropia. Igualmente, aunque la pregunta está propuesta en un lenguaje verbal y algebraico, se pretende observar si utilizan el registro gráfico para abordarla, dado que es más intuitivo caracterizar las funciones por su forma.

Las acciones esperadas en esta pregunta quedan reflejadas así:

\begin{tabular}{|c|c|}
\hline & $R_{H}, R_{F}$ \\
& $T_{F}$ \\
& $C_{H \rightarrow G}, C_{F \rightarrow G}, C_{G \rightarrow F}$ \\
& $C_{F \leftrightarrow G}, C_{G \leftrightarrow F}$ \\
& $P S_{G}$ \\
\hline
\end{tabular}

\section{Análisis y discusión}

Durante la entrevista con la alumna MA1 emergieron diversos obstáculos y dificultades que tiene esta alumna en relación con el concepto de límite y la diferencia entre serie y sucesión. En un primer momento, observamos que no realiza transferencia de los conceptos de sucesiones y comprobamos más adelante que sus concepciones relativas a las nociones de límite, sucesión y serie son inadecuadas; por ejemplo, la misma noción de convergencia le ofrece serias dificultades:

A: Pero no entiendo converger, porque no es... no es que sea... ¡Ay, Dios! [...] ... pues lo de converger está «fastidiao»». Pero no es como... como una suma. No es una suma, ¿no?

E: No, o sea... lo que tienes es... bueno, lo que tienes es primero la integral de uno a diez, que te da un número. Después la integral de uno a veinte, que sería el segundo término de la sucesión.

A: ¿Y qué es lo que tengo que ver para que converja?

Más adelante, escribe en la pizarra una integral genérica $\int_{1}^{\infty} f(x) d x$ y trata de clasificarla o aplicarle algún criterio (aparentemente, sabe algunos enunciados de memoria) sabiendo las condiciones que cumple. Por tanto, parece darle gran importancia al trabajo en el registro formal.

La alumna MA2 tiene también en esta pregunta diversas dificultades. Pensamos que, principalmente, se deben a la ausencia de significado de los términos relativos a series y sucesiones. Por ejemplo, si se le pregunta cuándo es convergente una sucesión, responde que cuando tiene límite. Sin embargo, si se le pregunta qué quiere decir tener límite, no sabe responder. Por tanto, las tareas de transferencia quedan interrumpidas por esta deficiencia, y las acciones $T_{F}$ no puede llevarlas a cabo correctamente.

La forma de enfrentarse a esta pregunta del alumno MA3 fue totalmente «formal» y muestra que las transferencias 
dentro del registro algebraico no le resultan muy difíciles. Sin embargo, cuando se le pide explícitamente que cambie al registro gráfico, este alumno no es capaz de coordinar los resultados e intuiciones que tenía formalmente:

E: ¿Y se te ocurre algo sobre la forma que tienen que tener las funciones? ¿Se te ocurre algo?

A: Buffff...

E: No la ecuación, sino la forma, o qué forma... si hay alguna forma que sí, o alguna forma que... que no.

[...]

A: No estoy en condiciones para... Pero... No sé, cuando son polinomios o... yo me atrevería a decir que... si un polinomio, eh... O no un polinomio, sino la inversa de un polinomio. [Señala a la familia $1 / x^{n}$ que ya tenía escrita.]

Finalmente, se escabulle del registro gráfico para pasar al algebraico. Aunque es capaz de señalar funciones que no, no sabe decir por qué, ni aporta alguna interpretación gráfica para que esto suceda.

El alumno MA6 abordó esta pregunta directamente en el registro gráfico, pero afirma:

E:[...] ¿Se te ocurre alguna caracterización de las funciones, alguna condición que tengan que cumplir?.... ¿crees que no se puede imponer nada...? [...]

A: Una función tal que el límite cuando $x$ tiende a infinito de $f(x)$ sea igual cero. [Mientras lo escribe.] $\operatorname{Lim} f(x)=0^{16}$. No sé... [Se encoge de hombros]

E: ¿Por qué?

A: Mm... hombre, estoy diciendo que se acerque mucho al cero. [Señala la asíntota de su curva.]

E: Ajá.

A: ...pero no sólo por eso. Yo qué sé, también una función que el límite sea cero no tiene por qué... por qué converger. Entonces sería una condición que sería... [Señala el límite que escribió.] interesante para el asunto.

Probablemente el hecho de pensar que es necesario que la función tienda a cero para que su integral converja venga provocado por el poco uso del registro gráfico y de contraejemplos adecuados durante el proceso de aprendizaje.

\footnotetext{
Pregunta 3

Calcula las siguientes integrales: $\int_{2}^{\infty} \frac{1}{x-1} d x \quad \pi \cdot \int_{2}^{\infty} \frac{1}{(x-1)^{2}} d x$

Interpreta geométricamente los resultados. ¿Hay alguna relación entre ambas integrales?
}

En esta pregunta los objetivos son los mismos que en la prueba escrita, es decir, ver cómo se enfrentan los estudiantes al cálculo de integrales impropias «sencillas» y enfrentarlos a un resultado paradójico, pero de forma encubierta, para analizar cuántos se dan cuenta de éste.

Las acciones esperadas son las siguientes:

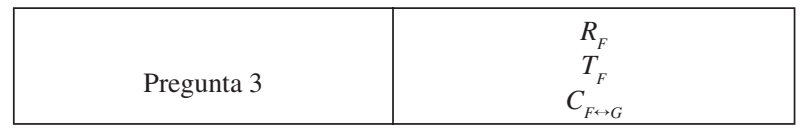

\section{Análisis y discusión}

La alumna MA1, al abordar la segunda integral, dice: «Esto es el volumen, ¿no?» y dice que lo sabe «porque es la fórmula». Sin embargo, luego no sabe exactamente con respecto a qué eje gira la función y concluye que da igual hacerla girar respecto de un eje que del otro, «porque es simétrica»: de donde se observa una nueva carencia de significación de los conceptos previos y en el uso del registro gráfico.

El entrevistador decide averiguar el alcance de su intuición o si ha dotado de significado a los ejemplos que se han resuelto en clase:

E: ¿Recuerdas lo que te dio la integral de antes?

A: Infinito...

E: Infinito. ¿Y qué representa ésta?

A: El volumen..

E: ¿Y, entonces, me puedes decir algo del resultado sin haberla hecho? O... ¿no me puedes decir nada?

A: Ah, que el volumen es infinito [... ] El volumen de una cosa infinita es infinito. Si tú tienes una cosa muy grande, muy grande, muy grande, y la haces girar, girar, girar... pues se irá expandiendo, por todos los lados... ¿no?

Observamos aquí el obstáculo que denominamos de ligación a la compacidad, ya que parece que la alumna sólo concibe un volumen como finito si la figura es cerrada y acotada. También creemos que nos encontramos con el obstáculo de homogeneizar dimensiones, consistente en creer que las propiedades de una figura en un número de dimensiones se mantienen al aumentar o disminuir este número; así, una figura de área infinita debería engendrar un volumen infinito (González-Martín, 2002).

La alumna MA2, para calcular, divide la integral:

$$
\int_{2}^{\infty} \frac{d x}{x-1}=\int_{2}^{3} \frac{d x}{x-1}+\int_{3}^{\infty} \frac{d x}{x-1}
$$

y se queda sólo con la primera de ellas, despreciando la segunda, pues es la primera integral «la que indica el carácter»y, si calculamos la primera integral, tenemos el problema resuelto. Esta forma de proceder es fruto de un error de interpretación de un criterio de convergencia ${ }^{17}$ y revela errores algebraicos y falta de coordinación entre los registros algebraico y gráfico, pues no se da cuenta de que está afirmando que el área en $[2, \infty)$ equivale al área en $[2$, 3 ). Cuando se le pide que explique un poco más su procedimiento para resolver la integral, explica que se usa para determinar su carácter; que se toma un valor cualquiera en el intervalo de integración $[2, \infty)$ y se separan las integrales, siendo la primera la que se toma. En sus palabras: «[...] tomé 3, pero podía haber tomado 35».

El alumno MA4 es de los diez alumnos que, en el cuestionario, enuncia que una integral representa el área y la 
otra, el volumen de la misma función; y es de los tres alumnos que enuncian claramente que el área puede ser infinita y el volumen, finito. Además, realiza correctamente la coordinación entre los registros formal y gráfico para interpretar geométricamente el resultado, aunque afirma que, aunque contradice la intuición, se fía de lo que le dicen las herramientas algebraicas:

A: He intentado ver lo que signifi... por qué eso es así, pero... Matemáticamente está correcto [Señala los cálculos.], pero... A la hora de verlo así, en la gráfica... Un poco raro... Raro es.

El obstáculo en el registro gráfico puede deberse a la tendencia a transferir las propiedades del área a las del volumen generado (que denominamos homogeneizar dimensiones).

\section{Pregunta 4}

¿Por qué el siguiente cálculo es obviamente erróneo?

$$
\int_{-1}^{1} \frac{d x}{x^{2}}=\int_{-1}^{1} x^{-2} d x=\left.\frac{x^{-1}}{-1}\right|_{-1} ^{1}=\left.\frac{-1}{x}\right|_{-1} ^{1}=\frac{-1}{1}-\frac{-1}{-1}=-2
$$

A esta cuestión, en el nivel académico de nuestros estudiantes, se le puede dar respuestas correctas razonando, al menos, de dos formas diferentes. En el registro algebraico, se ha calculado como una integral de Riemann, una función que presenta una discontinuidad esencial en el intervalo. Gráficamente también resulta obvio que el resultado es erróneo, ya que la función es estrictamente positiva $y$, sin embargo, se obtiene un resultado negativo para su integral.

Nuevamente, estamos interesados en ver qué registro es el elegido por los alumnos para enfrentarse a esta situación, a pesar de que la mayoría define la integral como un área.

Las acciones que se espera que los alumnos desarrollen son:

\begin{tabular}{|c|c|}
\hline & $R_{F}$ \\
Pregunta 4 & $T_{F}$ \\
& $C_{F \leftrightarrow G}, C_{G \leftrightarrow F}$ \\
& $P S_{G}$ \\
\hline
\end{tabular}

\section{Análisis y discusión}

La alumna MA1 utiliza un criterio de convergencia con el fin de calcular el valor de la integral, al igual que la alumna MA2 en la pregunta anterior. Ella dice que se puede proceder, en general, del siguiente modo:

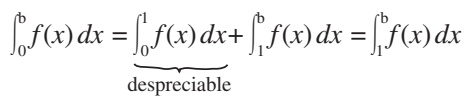

porque «hay un teorema que decía algo asî ${ }^{18}$ ».

En este caso, los errores cometidos se deben a una mala interpretación de las herramientas formales, usándolas en situaciones que no les corresponde.

\section{Pregunta 5}

La siguiente figura muestra el resultado de hacer girar la curva $y=e^{x}$ alrededor del eje $\mathrm{OX}$.

Si siguiéramos prolongando la figura hacia la izquierda, ¿qué volumen crees que encerraría?

Para comprobar tu intuición, lo calcularemos. Cada sección circular tiene un radio de $e^{x}$. Por tanto, el área de cada sección es:

$$
A(x)=\pi \cdot(\text { radio })^{2}=\pi \cdot\left(e^{x}\right)^{2}=\pi \cdot e^{2 x} .
$$

Si sumamos ahora todas las áreas, obtendremos:

$$
V(x)=\int_{-\infty}^{1} A(x) d x=\int_{-\infty}^{1} \pi \cdot e^{2 \mathrm{x}} d x=\pi \cdot \lim _{\mathrm{b} \rightarrow-\infty} \int_{\mathrm{b}}^{1} e^{2 \mathrm{x}} d x .
$$

¿Qué sucede? Interpreta el resultado.

Esta pregunta ofrece directamente un resultado paradójico a los alumnos. Sin embargo, en la prueba escrita pareció que algunos lo abordaban con total naturalidad $(32,25 \%)$. Se trata de un enunciado que, restringido al registro algebraico, puede no resultar tan paradójico; sin embargo, cuando se interpreta gráficamente, se muestra con toda su conflictividad.

Las acciones a desarrollar son las que aparecen en el siguiente cuadro:

\begin{tabular}{|c|c|}
\hline & $R_{H}, R_{G}, R_{F}$ \\
& $T_{F}$ \\
Pregunta 5 & $C_{H \rightarrow G}, C_{H \rightarrow F}, C_{F \rightarrow G}, C_{G \rightarrow F}$ \\
& $C_{F \leftrightarrow G}, C_{G \leftrightarrow F}$ \\
& $P S_{F}$ \\
\hline
\end{tabular}

\section{Análisis y discusión}

La alumna MA2 es un buen ejemplo de los conflictos cognitivos que genera esta cuestión; al interpretar el resultado, afloran los problemas.

A: Pero es que yo lo que no entiendo es eso. ¿Cómo... cómo podemos decir... que...? O sea, ¿cómo podemos darle un número a algo que es infinito... se supone... Eso es lo que nunca he entendido yo [...] Esta función es infinita, se supone, no está acotada, es infinita...

E: Ajá.

A: ...y me dicen que eso tiene un volumen de un número... [Mira al entrevistador y se encoge de hombros.] No lo entiendo por qué me puedes asegurar eso, ¿sabes?... Que no...

Observamos nuevamente la presencia del obstáculo que denominamos ligación a la compacidad. La alumna no puede siquiera concebir el procedimiento de calcular un área o un volumen de una figura de forma infinita. Pensamos que esto se relaciona con su incapacidad de concebir una integral con uno de los límites de integración varia- 
ble; incluso puede que sea el propio concepto de límite el que esté en juego. Opinamos que esto se puede deber a sus limitaciones también para asimilar procesos infinitos. Probablemente su concepción del infinito, incluso en los cálculos de límites, sea de un infinito potencial, al que nunca se puede llegar.

El alumno MA3 afirma con seguridad que se trata de una función con área infinita y volumen finito; no sabemos a qué se debe que considere que el área es infinita, pues afirma intuitivamente que, por ser una exponencial, el volumen será finito. El camino de lo gráfico a lo formal lo realiza con ausencia de algunos significados.

Pregunta 6
Sabemos que $\sum_{n=1}^{\infty} \frac{1}{n}=\infty$ y $\sum_{n=1}^{\infty} \frac{1}{n^{2}}=\frac{\pi^{2}}{6}$
En vista de estos resultados,
¿qué puedes decir del valor de $\int_{1}^{\infty} \frac{1}{x} d x$ y $\int_{1}^{\infty} \frac{1}{x^{2}} d x$ ?
Ayúdate de la gráfica adjunta.

Esta pregunta sólo es respondida de la forma esperada en la prueba escrita, esto es, estableciendo la relación explícitamente entre las integrales, las series y utilizando la gráfica adjunta, por un alumno de los 31 que rellenaron el cuestionario (el alumno MA5). Éste fue uno de los motivos para incluirla en la entrevista, ya que queríamos averiguar las razones por las que algunos alumnos no la habían abordado (incluso algunos con un rendimiento alto en el conjunto de la prueba). También nos preguntamos si, con alguna pista, los alumnos podrían establecer las relaciones precisadas.

En esta pregunta se aborda de forma explícita una relación entre series e integrales; nuevamente, es necesaria la combinación de los registros gráfico y algebraico para obtener toda la riqueza de esta relación.

Aparentemente, la gráfica había sido desechada por la gran mayoría de los alumnos participantes. Nos preguntamos también por qué se prefiere trabajar exclusivamente en el registro algebraico en una pregunta en que se pide de forma explícita que se use una gráfica; una de las dudas que se nos planteaba era si esto se debe a la no-transferencia del conocimiento sobre las series o a la no-adecuada transferencia del conocimiento sobre integración al registro gráfico.

Las acciones esperadas quedan resumidas como sigue:

\begin{tabular}{|c|c|}
\hline & $R_{G}, R_{F}$ \\
& $T_{F}, T_{G}$ \\
& $C_{F \rightarrow G}, C_{G \rightarrow F}$ \\
& $C_{F \leftrightarrow G}, C_{G \leftrightarrow F}$ \\
& $P S_{F}, P S_{G}$ \\
\hline
\end{tabular}

\section{Análisis y discusión}

La alumna MA1 no ve ninguna relación entre los sumatorios y las integrales a primera vista y luego piensa si tendrá que ver con la partición ${ }^{19}$. Más adelante comenta que la gráfica le dice que «esto sigue p'allá» (hacia más infinito). Por tanto, en un primer momento no reconoce la información proporcionada en el registro gráfico, y tampoco realiza transferencia ninguna de series a integrales.

Un poco después dice:

A: Pero las integrales se asocian con los límites, no con los sumatorios ésos...

De donde podemos inferir que su concepción de integral no está desarrollada del todo, además de que no realiza transferencias adecuadas.

Más adelante, vuelve a presentarse el obstáculo de homogeneizar dimensiones, consistente en suponer que las propiedades que tiene un área se «heredan» al volumen que ésta genera; en este caso, la infinitud. Pensamos que, en esta situación, el obstáculo va ligado directamente al que denominamos de ligación a la compacidad, pues es el hecho de que la figura tenga una forma infinita lo que hace que la alumna reaccione como lo hace:

A: No sé por qué... [Pausa, mirando las preguntas.] No sé por qué... sale esto... El área es infinita y el volumen es finito ${ }^{20}$ [Para ella.] ¿Pero el volumen no se supone que es más grande?... Son muchas áreas sumadas... [Pausa] No sé... Pues ya está [Sigue mirando ambas preguntas.]... Pero es que es eso, lo que te dije... ¿Por qué a veces se desprecia y a veces no?... No lo sé, no sé. [Mira al entrevistador.]

El comportamiento del alumno MA3 es significativo, pues rechaza expresamente la información dada por la gráfica; él mismo reconoce que no la utiliza en su desarrollo.

El alumno MA4 relaciona rápidamente las integrales con las sumas:

A: A ver, las integrales tienen que ver con el sumatorio... en que... la integral lo que hace es sumar. Sumar rectángulos. Infinitos rectángulos. E: Ajá.

A: Pues... Y por eso coinciden. Porque coinciden, ¿no? [Dice para sí; se queda leyendo el papel.]

Sin embargo, como observamos, el proceso de transferencia que realiza de su conocimiento sobre series a esta nueva situación no es del todo correcto, pues cree que el resultado de las sumas coincidirá con el valor de las integrales ${ }^{21}$.

En un primer momento, no es capaz de interpretar el significado de los rectángulos que aparecen en la gráfica. Por tanto, opinamos que, en este punto, el alumno no está coordinando la representación gráfica con la información algebraica de que dispone. Tras recibir alguna pista, trabaja de forma adecuada.

Esta pregunta es la única que el estudiante MA6 deja en 
blanco en el cuestionario. Ya durante la entrevista nos aclaró que se vio falto de tiempo y por eso no intentó nada en esta pregunta.

El estudiante reconoce que, en un primer momento, piensa que las integrales deben coincidir con el valor de las sumas, lo cual puede deberse a un proceso erróneo de transferencia. También comenta que se le ocurre abordar la pregunta algebraicamente, aunque prefiere buscar otro enfoque. Sin embargo, al enfrentarse a la segunda integral, realiza incorrectamente la transferencia del primer ejemplo al segundo. Intenta abordar la segunda integral como un volumen, por lo que necesita hacer girar también los rectángulos; no se da cuenta de que en éste conviene abordar ambas integrales en el plano. Ante la duda, prefiere volver al formalismo; tras alguna pista, hace un desarrollo similar al del primer caso.

\section{CONCLUSIONES Y RECOMENDACIONES}

\subsection{En relación con las preguntas de investigación}

Del análisis de los cuestionarios escritos se desprenden ciertas evidencias de que los alumnos, para trabajar, prefieren enunciados claros y directos, en el registro algebraico, a pesar de las deficiencias que muchos ofrecen en este registro. Las preguntas de razonamiento y aquéllas en las que se pedía una interpretación de los resultados no fueron abordadas satisfactoriamente en general. Los alumnos prefieren realizar cálculos algebraicos a interpretar resultados.

Se observa que los estudiantes se quedan desconcertados cuando se les pide que definan el concepto de integral definida. Y no sólo eso, sino que este hecho parece confundir a algunos. Muchas veces recurren expresamente a su memoria, y la frase «no me acuerdo» se repite a lo largo de las entrevistas. Los alumnos prefieren enunciados de tipo algorítmico y con instrucciones claras de lo que se les pide. Además, cuando las cuestiones no algorítmicas presentan otro registro de representación (en nuestro caso, el gráfico) producen grandes dificultades en los alumnos, que no están acostumbrados a trabajar en este registro (Calvo, 1997). De esta manera, preguntas que requieren trabajar explícitamente en un registro distinto del algebraico son abandonadas por un gran número de estudiantes.

Las entrevistas evidencian que el empleo de gráficas para ayudarse en sus razonamientos no es una herramienta demasiado habitual. Además, en algunas ocasiones los estudiantes se resisten a explicitar las representaciones gráficas.

El estudio, aunque de naturaleza distinta, confirma los resultados de Orton (1983) con respecto a la preponderancia del pensamiento algebraico en los alumnos. Aunque el registro algebraico ocasione grandes dificultades a veces, es en el que están acostumbrados a trabajar. Esto se desprende por su tendencia a usarlo incluso en cues- tiones de más fácil respuesta en el registro gráfico o en cuestiones de respuesta meramente verbal.

Algunos estudiantes ni siquiera reconocen el registro gráfico como un registro de trabajo matemático, lo que provoca en algunos casos su incapacidad para trabajar en él. Sin embargo, el uso de este registro de forma más activa durante la instrucción y en los ejercicios y problemas propuestos a los alumnos podría paliar este tipo de carencias. Aunque los alumnos a veces hagan interpretaciones de sus resultados, no están acostumbrados a explicitarlas, por lo que este tipo de cuestiones les ofrece inseguridad. Por otro lado, tal como se ha visto, muchas veces no tienen claro el paso de lo algebraico a lo gráfico, lo que impide establecer conexiones entre el resultado obtenido y la interpretación gráfica.

Nuestros resultados también confirman los expuestos por Eisenberg y Dreyfus (1991) en contextos diferentes, que también registran una clara reticencia de los alumnos a usar el registro gráfico, que requiere demandas cognitivas mayores.

En consecuencia, el hecho de que no suelan interpretar gráficamente los resultados que obtienen es un impedimento para que sean conscientes de algunos resultados paradójicos a los que se llega. En el cuestionario no queda claro si los alumnos son o no conscientes de estos resultados o si, simplemente, por resultarles desconcertantes prefieren evitar comentarlos. Por el contrario, en las entrevistas vemos cómo estos resultados les ofrecen problemas que en algunos casos constituyen verdaderos obstáculos. Algunos estudiantes se enfrentan a ellos apoyándose en el registro algebraico, en el que confían ciegamente. Se observa igualmente que los alumnos con menores dificultades en las tareas de conversión parecen ser los que menos desconcierto muestran ante este tipo de cuestiones.

El uso de razonamientos intuitivos, tanto algebraicos como gráficos, está relacionado con el nivel de comprensión que se tiene de los conceptos que entran en juego, por lo que alumnos con grandes lagunas no suelen disponer de estos razonamientos.

Las entrevistas ponen de nuevo de manifiesto que la gráfica en el enunciado de la pregunta 6, en general, no aporta nada a los alumnos entrevistados. Éstos no saben cómo utilizar la información en ella mostrada, lo que revela nuevamente una ausencia de coordinación entre el registro gráfico y el algebraico. Se pone de manifiesto que el paso de lo algebraico a lo gráfico ocasiona menos dificultades que el proceso inverso.

Una correcta comprensión del concepto de integral impropia necesita visualizar el cálculo de áreas como un proceso dinámico, ya que esta visión permite concebir la función integral y calcular su límite. Tall (1992b) registra que las diferentes representaciones de las funciones ocasionan dificultades a los alumnos. Con más motivo encontramos ausencia de coordinación entre representaciones al abordar el concepto de integral impropia, que requiere del de función. Y coincidimos con Calvo (1997) 
en que el hecho de visualizar e integrar diferentes representaciones de un concepto no es algo que el alumno realice por sí solo, sino que se debe aprender a hacer.

Las entrevistas muestran las deficiencias de algunos alumnos en conceptos como el de límite, que están bien presentes en el tema de la integración impropia. Esto confirma resultados como los expuestos por Artigue (1995) y Tall (1992a, 1992b). También el mismo concepto de integral definida ofrece varias dificultades, incluso en alumnos de nivel alto. El hecho de aprenderla como un área genera un obstáculo en algunos casos, pues se induce que siempre dará un valor positivo.

También queda clara la falta de transferencia de unos conceptos a otros. Por ejemplo, nos encontramos con alumnos que no entienden por qué a veces se divide una integral impropia en dos y una de ellas se desprecia. Además de no tener claros los criterios y su uso, refleja una ausencia de conexiones con la misma forma de proceder en el caso de las series numéricas.

\subsection{En relación con las dificultades, obstáculos y errores}

Una de las principales dificultades que observamos proviene directamente de la ausencia de significado de herramientas fundamentales para comprender este concepto, como el uso de límites, la misma noción de convergencia, la definición de integral definida y algunas definiciones básicas de series y sucesiones (estudiantes MA1, MA2 y MA4). La noción de convergencia y algunos conocimientos mínimos sobre sucesiones y series (cómo una suma infinita puede dar finito, por ejemplo) emergen como conocimientos previos, sin los cuales difícilmente se podrá adquirir adecuadamente una comprensión del concepto de integral impropia.

También la falta de coordinación entre registros o el no-reconocimiento de uno de los registros, como en el alumno MA3, originan dificultades a los estudiantes (MA4 y MA6) que aprenden los conceptos relacionados con la integración impropia. Ya hemos comentado cómo algunas contradicciones les hacen dudar y cómo el uso del registro gráfico en el enunciado de las preguntas produce un alto índice de abandono.

Un obstáculo que hemos registrado es el generado por la concepción errónea de que la integral definida es siempre un área y, por tanto, ha de dar un valor positivo (alumnos MA1, MA2, MA3 y, en menor medida, MA6). Este obstáculo ha sido detectado también por otros autores en otros contextos (Calvo, 1997; Turégano, 1998; Bezuidenhout y Olivier, 2000). Además, en algunos casos apreciamos una concepción exclusivamente operativa de la integral definida.

Otro obstáculo es el producido por el empleo de una concepción estática de los procesos límite, además del uso del infinito potencial en lugar del actual (alumnos MA1 y MA2). En algunos casos, hemos detectado una concepción del límite como una mera operación algebraica, lo que puede dificultar la conceptualización del cálculo del área de una figura de aspecto infinito.

Un obstáculo que aflora naturalmente en algunos resultados contradictorios del cálculo de integrales impropias es el que hemos denominado de ligación a la compacidad (MA1 y MA2). Este obstáculo parece estar determinado por la ausencia de coordinación entre el registro gráfico y el algebraico, además de por la falta de ejemplos y contraejemplos en el campo de las series numéricas.

El obstáculo ya mencionado de «homogeneizar dimensiones ( MA1) consiste en atribuir a un volumen las propiedades del área que lo genera por revolución. Parece nuevamente que este obstáculo se debe, entre otros factores, a la ausencia de coordinación entre registros. Estos dos obstáculos, relacionados entre sí, parecen también relacionados con el obstáculo de «heterogeneidad de las dimensiones» (Schneider, 1991). Una de las manifestaciones de éste consiste en ver un volumen como una superposición de superficies: «Creo que es el método más intuitivo que se puede imaginar, pues siempre he imaginado un volumen como una superposición de superficies. Por medio del estudio de las dos primeras dimensiones podemos encontrar la tercera, o un volumen es una superficie multiplicada por una altura.» Una de sus consecuencias lleva a considerar que iguales perímetros encierran áreas iguales y que iguales áreas llevan a igual volumen.

De este modo, si concebimos el volumen como compuesto de distintas láminas y se sabe que el área de la lámina «generatriz» (cuando se trata de un volumen de revolución) es infinita, se concluye que el volumen lo será también.

Schneider relaciona también este obstáculo con los problemas que ocasiona el uso del infinito actual. Nuevamente, si el alumno concibe el volumen como un agregado de secciones planas, atribuirá al resultado (el volumen sería el límite del agregado de áreas) las propiedades de los elementos (las secciones); nuevamente el volumen se concibe como forzosamente infinito. Esto último se describe en Tall (1992a, 1992b).

En el caso de las integrales de funciones como $1 / x$ y $1 / x^{2}$, los alumnos las interpretan siempre como área y volumen de una misma función. Pero, cuando se trabaja en el plano, parecen incapaces de ver ambas integrales como áreas (alumno MA6 en la pregunta 6). Se trata de un obstáculo que parece nuevamente ligado a la coordinación entre registros, pero, en este caso, a la no-flexibilidad en la coordinación.

Quizá sean necesarias actividades donde se trabajen con este tipo de situaciones y donde se conjugue el trabajo en dos y tres dimensiones.

Entre los errores analizados, destacamos también la carencia de significado al malinterpretar los enunciados de algún teorema o criterio y utilizarlo en otro caso (MA1 y MA2). Este tipo de actuaciones puede llevar a resultados totalmente incoherentes. Por ejemplo, en el criterio de tomar sólo la 
«cola» de la integral para determinar su carácter, algunos estudiantes acaban pensando que es ésta la que condensa el valor de la integral, por lo que acaban calculándola en un subintervalo de integración pensando que obtendrán el valor de la integral total. Claramente, esta forma de actuar revela también una falta de coordinación entre los registros gráfico y algebraico, además de deficiencias en la definición de integral definida.

Otros errores también registrados son de problemas de sintaxis puramente algebraicos. Algunos se deben a carencias de significado de los alumnos en el uso de las propiedades de las operaciones aritméticas (alumna MA2) y otros los hemos considerado meramente casuales.

\subsection{En relación con el modelo de competencia}

Después de analizar todas las entrevistas de los alumnos, se hizo un análisis de las acciones realizadas y no realizadas en todas las preguntas de la entrevista, obteniendo un paisaje global de su desempeño, que permitió la clasificación de éstos en las categorías de nuestro modelo de competencia, atendiendo al número total de acciones realizadas:

\begin{tabular}{|l|c|c|c|c|c|c|}
\hline Alumno & MA1 & MA2 & MA3 & MA4 & MA5 & MA6 \\
\hline Categoría & 1B-2A & 1A-1B & 2A & 2B & 3A & 2A-2B \\
\hline
\end{tabular}

El modelo de competencia diseñado nos puede ayudar a analizar explícitamente las acciones que los alumnos realizan en relación con las que se espera que lleven a cabo cuando entran en juego los registros de representación gráfico y algebraico en el aprendizaje de los conceptos referentes a la integración impropia.

Es necesario, sin embargo, concretarlo aún más al caso de dos registros de representación, pues ha sido adaptado directamente de otro trabajo en lenguaje algebraico (Socas, 2001). Esto implica resaltar las especificidades de nuestro estudio particular.

A pesar de ello, opinamos que el modelo de competencia utilizado en este estudio nos ha resultado extremadamente útil para realizar una primera aproximación al análisis de la comprensión de los alumnos de la integral impropia. Además, nos ha permitido examinar e identificar algunas dificultades y obstáculos que nuestros alumnos presentan.

Será motivo de posteriores trabajos el refinamiento de las categorías diferenciadas en nuestro modelo, además del diseño y selección de tareas que nos ayuden a evaluarlo.

\section{ALGUNAS CUESTIONES ABIERTAS}

Una de las principales cuestiones que nos queda por resolver es qué sucede cuando entra en juego el registro numérico en las tareas que se exponen. Aunque utilizamos este registro en una de las cuestiones propuestas en el cuestionario, pensamos que su formulación produjo un tipo de respuestas muy simples que puede no resultar muy fiable.
En posteriores experiencias habrá que plantearse qué actividades deben resolver los alumnos para que entre en juego este registro y también cómo nos pueden ayudar éstas a caracterizar el nivel de comprensión de nuestros estudiantes. El estudio del desempeño de los alumnos cuando interactúan estos tres registros de representación (algebraico, gráfico y numérico) y el tipo de conversiones y coordinaciones que pueden realizar es, sin lugar a dudas, un trabajo de mucha más envergadura que éste. Sin embargo, será importante plantear preguntas de investigación similares que utilicen por separado los distintos registros de representación.

Otra cuestión que emerge del estudio realizado es la de profundizar en los obstáculos y dificultades aquí caracterizados y localizados. Nos parece significativa la labor de identificación más sistemática de errores, dificultades y obstáculos de nuestros alumnos en el aprendizaje de los conceptos específicos de la integración impropia, tratando de relacionarlos con otras investigaciones previas y relacionarlos con otros tópicos matemáticos. Resultaría interesante una comparación, por ejemplo, entre los obstáculos y dificultades detectados alrededor de la noción de límite o de integral definida y los que se detecten relacionados con la integración impropia.

Como hemos indicado en este trabajo, nos interesábamos más por un problema de aprendizaje que de enseñanza y es en este sentido en el que aparece otra cuestión que nos parece fundamental. Una vez detectados diversos obstáculos y dificultades que los alumnos presentan al aprender el concepto de integral impropia y presentado un primer análisis del nivel de comprensión de este objeto como concepto y herramienta de cálculo, nos parece que llega el momento de plantearse qué hacer en la práctica con su enseñanza.

La labor de diseñar una secuencia de enseñanza-aprendizaje donde se explicite el uso de los distintos registros y se trabaje con ejemplos y contraejemplos se presenta no sólo interesante, sino también importante. Nos preguntamos, además: ¿Sería factible que esta secuencia estuviera basada en el uso de un CAS (Computer Algebra System), usado como mediador en el proceso de enseñanza-aprendizaje del objeto integral impropia? Es en esta labor en la que nos encontramos en la actualidad.

\section{NOTAS}

* Este trabajo ha sido parcialmente financiado por la Beca AP20002106 y el proyecto DGI BXX2000-0069 del MCT.

${ }^{1}$ En particular, nuestra experiencia se centra en alumnos de la titulación de Matemáticas.

${ }^{2}$ Suele ser la integral que se estudia en los primeros niveles universitarios, en general, y la que se introduce normalmente en el bachillerato.

${ }^{3}$ Un contraejemplo clásico viene dado por la función de Dirichlet.

${ }^{4}$ Esta pregunta coincide con nuestra primera pregunta, tanto del cuestionario como del protocolo de las entrevistas.

${ }^{5}$ Aunque, en nuestra opinión, sus respuestas son dudosamente correctas. Cuatro alumnos la definen como «el área entre la gráfica y el eje $x$ entre $x=a$ y $x=b »$, que no tiene en cuenta los cambios de signo de la 
función; y tres como $\int_{a}^{\mathrm{b}} f(x) d x=F(b)-F(a)$, definición operativa y que obvia varias condiciones.

${ }^{6}$ En el sentido dado al término en Monaghan y otros (1999) y González-Martín y Camacho (2004).

${ }^{7}$ Definida ésta como la aprehensión o la producción de una representación semiótica.

${ }^{8}$ De esta forma, no se consideran evoluciones en el uso del registro algebraico (1- el estudiante usa los signos nuevos con el significado de los antiguos, 2- estructura el nuevo sistema con la organización del antiguo; y 3 - los signos actúan con significados propios), sino evoluciones en el uso de dos registros (1- el estudiante usa un registro de forma incoherente, 2- se comienza el trabajo en un registro y las primeras coordinaciones con otro; 3 - se articulan los dos registros).

${ }^{9}$ En lo que sigue, nosotros usaremos el término algebraico, aunque mantenemos el subíndice $F$ por continuidad con otros trabajos de otros autores donde se usa esta notación.

${ }^{10} \mathrm{O}$ task-based interview, término usado en la literatura anglosajona. Véase, por ejemplo, Goldin (2000).

${ }^{11}$ Nos parecen importantes no sólo los comportamientos verbales, sino también los no verbales, para realizar inferencias sobre el pensamiento matemático y el aprendizaje de los entrevistados.

${ }^{12}$ Esta categorización se basa en la puntuación que se dio a cada prueba para que el profesor de la asignatura pudiera tener en cuenta los resultados de ésta en la nota final de la asignatura.

${ }^{13}$ Los fragmentos de entrevista escogidos lo han sido por considerarse que ilustran algunas de las dificultades, obstáculos y errores de nuestra primera clasificación. Para un análisis y comparación más exhaustivos entre las respuestas obtenidas en las seis entrevistas, consultar González-Martín (2002).

${ }^{14}$ Incluso al final de la entrevista se le propone una integral muy sencilla de una función lineal que cambia de signo en el intervalo de integración, con resultado negativo. Cuando ve este resultado, decide separarla en dos y cambiarle el signo a la integral negativa, para obtener un área positiva.

${ }^{15}$ El proceso cognitivo por el cual los procesos se conciben y se vuelven objetos manipulables se denomina encapsulación.

${ }^{16}$ Sin embargo, esta condición, aunque no es suficiente, ni siquiera resulta ser necesaria. Pero opinamos que, en este nivel, es la idea más intuitiva para los alumnos.

${ }^{17}$ En particular, el criterio afirma: $«$ Sea $a>0, f$ localmente integrable en $[a, \infty)$ y $f(x) \geq 0, \forall x \in[a, \infty)$. Sea $\lambda=\lim _{x \rightarrow \infty} x^{k} . f(x)$. Entonces. si, $\lambda \neq \infty \wedge$ $k>1 \Rightarrow \int_{a}^{\infty} f(x) d x$ converge; si $\lambda \neq 0 \wedge k \leq 1 \Rightarrow \int_{a}^{\infty} f(x) d x$, diverge». Para utilizar este criterio, si se tiene una integral que comienza en 0 , se separa en dos y se aplica el criterio a la segunda de ellas.

${ }^{18}$ Vuelve a hacer referencia al criterio nombrado anteriormente.

${ }^{19}$ Observamos, en general, una tendencia de los alumnos a identificar la serie asociada a una función $\left(f(1)+f(2)+f(3)+\ldots=\sum_{n=1}^{\infty} f(n)\right)$ con sus sumas de Riemann y, por tanto, con su integral.

${ }^{20} \mathrm{Al}$ comparar los valores de $\int_{1}^{\infty} \frac{1}{x} d x$ y $\int_{1}^{\infty} \frac{1}{x^{2}} d x$.

${ }^{21} \mathrm{Al}$ igual que la estudiante MA1, identifica la serie asociada a una función con sus sumas de Riemann y, por tanto, con su integral. 


\section{REFERENCIAS BIBLIOGRÁFICAS}

ARTIGUE, M. (1995). La enseñanza de los principios del cálculo: problemas epistemológicos, cognitivos y didácticos, en, Gómez, P. (ed.). Ingeniería didáctica en educación matemática, pp. 97-140. México: Grupo Editorial Iberoamericano.

BEZUIDENHOUT, J. y OLIVIER, A. (2000). Students' conceptions of the integral, en Nakahara, T. y Koyam, M. (eds.). Proceedings of the $24^{\text {th }}$ Conference of the International Group for the Psychology of Mathematics Education, vol. 2. Japón.

CALVO, C. (1997). «Bases para una propuesta didáctica sobre integrales». Tesis de maestría. Universitat Autònoma de Barcelona.

CAMACHO, A. y AGUIRRE, M. (2001). Situación didáctica del concepto de límite infinito. Análisis preliminar. Revista Latinoamericana de Investigación en Matemática Educativa (RELIME), 4(3), pp. 237-265.

DUVAL, R. (1993). Registres de représentation sémiotique et fonctionnement cognitif de la pensée, Annales de Didactique et de Sciences Cognitives, 5, IREM de Strasbourg, pp. 37-65.

DUVAL, R. (1995). Sémiosis et Penseé Humaine. Registres sémiotiques et apprentissages intellectuels. Neuchatel: Peter Lang.

EISENBERG, T. y DREYFUS, T. (1991). On the Reluctance to Visualize in Mathematics, en Zimmermann, W. y Cunnigham, S. (ed.). Visualization in Teaching and Learning Mathematics, pp. 25-37. Washington.

GARBIN, S. (1998). «Esquemas conceptuales e incoherencias de estudiantes de bachillerato en relación con el concepto de infinito actual contextualizado en problemas expresados en diferentes lenguajes matemáticos: verbal, geométrico, gráfico, algebraico y numérico. Estudio exploratorio». Tesis de maestría. Universitat Autònoma de Barcelona.

GOLDIN, G. A. (2000). A Scientific Perspective on Structured, Task-Based Interviews in Mathematics Education Research, en Kelly, A. E. y Lesh, R. A. (eds). Handbook of Research Design in Mathematics and Science Education. Londres: Lawrence Erlbaum Associates, pp. 517-546.

GONZÁLEZ-MARTÍN, A. S. (2002). «Dificultades, obstáculos y errores en el aprendizaje del concepto de integral impropia» Tesis. Universidad de La Laguna.

GONZÁLEZ-MARTÍN, A. S. y CAMACHO, M. (2002a). The improper integral. An exploratory study with First-Year University students, Proceedings of the Second International Congress on the Teaching on Mathematics. Grecia: J. Wiley.

GONZÁLEZ-MARTÍN, A. S. y CAMACHO, M. (2002b). The comprehension of the concept of improper integral in Mathematics students: representation and transference, en Giménez, J., Hahn, C. y Fitzsimons, G. (eds.). Proceedings of the $54^{\text {th }}$ CIEAEM Conference. Granada: Proyecto Sur (por aparecer).
GONZÁLEZ-MARTÍN, A. S. y CAMACHO, M. (2004). What is students' actual understanding about improper integration? International Journal of Mathematical Education in Science and Technology, 35(1), pp. 73-89.

HITT, F. (2000a). Construcción de conceptos matemáticos $y$ de estructuras cognitivas, segunda versión del artículo presentado en el Working Group: Representations and mathematics visualization del PME-NA, pp. 131-147. Tucson, Arizona.

HITT, F. (2000b). El concepto de infinito: obstáculo en el aprendizaje de límite y continuidad de funciones, Matemática Educativa: Aspectos de la Investigación Actual, sobre el XXV Aniversario del Departamento de Matemática Educativa del CINVESTAV-IPN. México DF.

MONAGHAN, J., JOHNSON, P., BERRY, J. y MAULL, W. (1999). Routine questions and examination-performance. Proceedings of the $23^{\text {rd }}$ International Conference for the Psychology of Mathematics Education (PME23). Israel. 2, pp. 105-112.

ORTON, A. (1983). Students' understanding of integration, en Educational Studies in Mathematics, 14(1), pp. 1-18. Holanda.

RASSLAN, S. y TALL, D. (2002). Definitions and images for the definite integral concept, en Cockburn y Nardi, (eds.). Proceedings of the $26^{\text {th }}$ International Conference for the Psychology of Mathematics Education (PME26), vol. 4, pp. 89-96. Norwich (UK).

SCHNEIDER, M. (1991). Un obstacle épistémologique soulevé par des «découpages infinis» des surfaces et des solidez. Recherches en Didactique des Mathématiques, 11(2-3), pp. 241-294.

SOCAS, M. (1997). Dificultades, obstáculos y errores en el aprendizaje de las matemáticas en la educación secundaria, en Rico, L. et al. (eds.). La educación matemática en la enseñanza secundaria, pp. 125-154. Barcelona: Horsori.

SOCAS, M. (2001). Investigación en didáctica de la matemática vía modelos de competencia. Un estudio en relación con el lenguaje algebraico. Departamento de Análisis Matemático. Universidad de La Laguna (sin publicar).

TALL, D. (1992a). Students' Difficulties in Calculus, Plenary presentation in Working Group 3, ICME. Québec.

TALL, D. (1992b). The transition to Advanced Mathematical Thinking: Functions, Limits, Infinity and Proof, en Grouws, D. A. (ed.). Handbook of Research on Mathematics Teaching and Learning, pp. 495-514. Nueva York: MacMillan Publishing Company.

TALL, D. (1994). Understanding the Processes of Advanced Mathematical Thinking, invited ICMI lecture at the International Congress of Mathematicians. Zurich.

TURÉGANO, P. (1998). Del área a la integral. Un estudio en el contexto educativo. Enseñanza de las Ciencias, 16(2), pp. 233-249. 